\title{
A PRÁTICA PEDAGÓGICA DO ENSINO DE CIÊNCIAS NAS ESCOLAS PÚBLICAS DE SANTA CRUZ - RN
}

Falconiere Leone Bezerra de Oliveira

Ciências e Educação Matemática, IFRN, Santa Cruz, Programa de Iniciação científica, Aluno do curso de Especialização em Geografia do Semi-árido. E-mail:

falconi_rn@yahoo.com.br.

Josiel Moreira da Silva

Ciências e Educação Matemática, IFRN, Santa Cruz, Bolsista do Programa de Iniciação Científica, Aluno do Curso de Licenciatura em Física. E-mail: josiel1nico@ hotmailcom.

Lauricéia Lays Santos Valença

Ciências e Educação Matemática, IFRN, Santa Cruz, Bolsista do Programa de Iniciação

Científica, Aluno do Curso de Licenciatura em Física.

E-mail: lauriceia_lays@ @otmail.com.

Janielle Gomes Freire

Ciências e Educação Matemática, IFRN, Santa Cruz

Aluna do curso de Especialização em Psicopedagogia - IESP

E-mail: janifreire@ hotmail.com

Leandro Silva Costa

Ciências e Educação Matemática, IFRN, Santa Cruz, Programa de Iniciação Científica, Professor de Biologia. E-mail: Leandro.costa@ifrn.edu.br.

\section{RESUMO}

O presente trabalho foi desenvolvido em cinco escolas públicas (Escolas Estadual Pedro Severino Bezerra, Escola Estadual José Bezerra Cavalcante, Escola Estadual Isabel Oscarlina Marques, Escola Estadual Francisco de Assis Dias Ribeiro e Escola Estadual Quintino Bocaiuva ) localizadas no município de Santa Cruz/RN. Através desta pesquisa, nós objetivamos pontuar as principais dificuldades encontradas pelos docentes da região para o desenvolvimento da prática pedagógica adequada. Nós pudemos perceber que grande parte dos professores entrevistados apresenta formação superior completa $(85,7 \%)$, o que teoricamente torna-os habilitados a lecionarem os conteúdos de ciências de uma forma mais apropriada. Com relação aos recursos utilizados pelos professores para o desenvolvimento das práticas de ensino, percebe-se que todos os professores entrevistados utilizam o quadro negro e livros didáticos em detrimento de aulas de campo e textos científicos. Além de a estrutura física ter sido considerada um empecilho para o desenvolvimento de aulas práticas, outros fatores são levados em consideração pelos professores, como falta de tempo para o preparo das aulas e domínio do conteúdo a ser abordado. Por fim, tendo em vista a grande dificuldade de professores para o desenvolvimento das aulas de ciências, nós decidimos verificar quais são as principais ferramentas utilizadas no planejamento destas, destacando-se os livros didáticos, citados por todos os professores, bem como internet, livros, revistas e periódicos. Os resultados encontrados neste trabalho tornam urgentes e necessários uma intervenção a fim de oferecer condições adequadas aos professores de ciências e consequentemente aos alunos, que passam a assimilar o conteúdo com uma maior facilidade.

PALAVRAS-CHAVE: Ciências da natureza; Livros didáticos; aulas práticas. 
THE PRACTICE OF SCIENCE TEACHING IN PUBLIC SCHOOLS OF SANTA CRUZ/RN

\begin{abstract}
This study was conducted in five public schools (Pedro Severino Bezerra, José Bezerra Cavalcante, Isabel Oscarlina Marques, Francisco de Assis Dias Ribeiro e Quintino Bocaiuva) located in Santa Cruz/RN. We object to shown the main difficulties encountered by teachers in the region for the development of appropriate pedagogical practice. We observed that the most teachers interviewed presents complete higher scholl $(85.7 \%)$, which theoretically creates the qualification to teach the most appropriate science. In respect to resources used by teachers to develop the teaching, it is clear that all use the blackboard and textbooks at the expense of scientific texts. In addition, the physical structure has been considered a primer obstacle to development of practical lessons, and other factors are taken into account by teachers, including a few time to prepare lessons and knowledge of the content. Finally, considering the difficulty of teachers for the development of science, we decided to prove what are the main tools used in the planning, that were: textbooks, cited by all teachers; internet; magazines and periodicals. The findings of this study shown that is necessary and urgent an intervention to provide appropriate conditions for science teachers and students, skill up the assimilation of the contents.
\end{abstract}

KEY-WORDS: Natural sciences; Textbooks; Practical lessons 


\section{A PRÁTICA PEDAGÓGICA DO ENSINO DE CIÊNCIAS NAS ESCOLAS PÚBLICAS DE SANTA CRUZ - RN}

\section{INTRODUÇÃO}

O ensino de ciências está sendo subjugado ao longo dos tempos, pois a concepção de ensino não é a mera transmissão de conhecimento e sim o compartilhamento do mesmo, assim segundo Paulo Freire "(...) ensinar não é transferir conhecimentos, mais criar possibilidades para sua produção ou sua construção" (FREIRE, 2000, p.25)

O ensino de ciências historicamente não compartilhava das idéias freiriana de compartilhar conhecimentos, mas sim a de expor. “(...) o cenário escolar era dominado pelo ensino tradicional, ainda que esforços de renovação estivessem em processo. Aos professores cabia a transmissão de conhecimentos acumulado pela humanidade.” (BRASIL, 1997.p.19)

Esse acúmulo de conhecimento era exposto ao aluno em grande escala, pois a qualidade do ensino era medida pela quantidade de matéria exposta e as avaliações eram baseadas em questionários que os alunos eram obrigados a responder, para que os seus conhecimentos fossem medidos.

A educação vem evoluindo em tempos e tempos e em sociedades diversas, ficando evidente que "somos seres históricos, já que nossas ações e pensamentos mudam no tempo, à medida que enfrentamos problemas não só da vida pessoal, como também das experiências coletivas" (ARANHA, 2006, p.19). Ainda, segundo Morin:

\footnotetext{
“A educação deve favorecer a aptidão natural da mente em formular e resolver problemas essenciais e, de forma correlata, estimular o uso total da inteligência geral. Este uso total pede o livre exercício da curiosidade, a faculdade mais expandida e a mais viva durante a infância e adolescência, que com frequiência a instrução extingue e que, ao contrário se trata de estimular ou, caso esteja adormecida de despertar" (MORIN, 2006, p.39).
}

Neste sentido, o ensino de ciência deve compelir as mais diversas formas nas mais diversas idades, pois "Para o ensino de Ciências Naturais é necessário a construção de uma estrutura geral da área que favoreça a aprendizagem significativa do conhecimento histórico acumulado e a formação de uma concepção de Ciência, suas relações com a Tecnologia e com a Sociedade". (BRASIL, 1997. P31)

Assim, segundo Piletti (2000, p.265), "O estudo das Ciências para o educando consiste em descobrir seu mundo, a fim de conhecê-lo, esclarecer suas dúvidas e valorizar o ambiente que o cerca". Dessa forma, o ensino de Ciências tem se preocupado em construir nos educandos o significado que a vida tem para cada um de nós. "A pessoa que lida com coisas da Ciência, que investiga que observa que é orientada nas suas observações, que recebe livros para pesquisar, torna-se diferente da que não tem essas oportunidades. Ela adquire uma força intelectual poderosa" (PILETTI, 2000, p.265)

O professor deve favorecer condições para que os seus alunos possam pesquisar, criar hipóteses e observar, mesmo que a sua instituição de ensino não favoreça condições adequadas para o ensino. Cabe a cada docente utilizar a sua criatividade para que o ensino das Ciências naturais aconteça. 
"Quando uma criança pesquisa, quando formula hipóteses, observa, experimenta, quando aprende a deixar a Natureza falar, permitindo-lhe responder com simplicidade às suas perguntas, começa a entender as relações entre o meio e o ser vivo, aprendendo a ser mais paciente, mais humilde, mais tolerante, mais responsável e também mais capaz de aprender." (PILETTI, 2000, p.265)

Visualizando essa ideia e concebendo-a as escolas, fica evidente que com a criatividade docente o mesmo pode melhorar a concepção de ensino-aprendizagem dos seus alunos para que a assimilação ativa dos conhecimentos referente ao ensino de Ciências aconteça.

Baseado nesses dados, e com o intuito de verificar como está sendo explorado o ensino de ciências nas escolas públicas do município de Santa cruz-RN, nós desenvolvemos este trabalho, o qual abordou como eixo temático principal a prática pedagógica do ensino de ciências.

Esta pesquisa tornou-se pertinente pelo fato de poder viabilizar novas formas de ensino de ciências, que podem vir a ser desenvolvidas nas escolas públicas de Santa Cruz - RN, podendo servir de instrumento para subsidiar melhorias no ensino de ciências nas escolas objetos de estudo.

\section{METODOLOGIA}

Neste trabalho, foi executada uma pesquisa exploratória, pois foi realizado um levantamento das informações sobre o nosso objeto de estudo $(\mathrm{O}$ ensino de Ciências nas escolas públicas de Santa Cruz). Também foi utilizada a pesquisa do tipo explicativa uma vez que analisamos o fenômeno estudado, os seus "porquês" e seus determinantes. Por fím, foi realizado um estudo de campo (Escolas) para buscarmos os dados diretamente no local, com auxilio de questionários estruturados destinados aos docentes. A escolha do questionário se deu pela objetividade das perguntas, o que nos ajudou a obter dados estatísticos com maior precisão. Além disso, vale ressaltar que o anonimato dos informantes (os professores), foi preservado.

As escolas foram selecionadas de acordo com os seguintes critérios: Existência da disciplina de ciências na matriz curricular; Presença de professores efetivos na escola; Autorização prévia dos diretores da escola. Portanto, foi possível desenvolver a pesquisa nas seguintes escolas: Escola Estadual Pedro Severino Bezerra, Escola Estadual José Bezerra Cavalcante, Escola Estadual Isabel Oscalina Marques, Escola Estadual Francisco de Assis Dias Ribeiro e Escola Estadual Quintino Bocaiuva.

Foram utilizados como eixos norteadores de nossa pesquisa os seguintes temas: Os professores apresentam formação acadêmica adequada? Quais os recursos utilizados no desenvolvimento das práticas docentes? Quais os recursos utilizados no desenvolvimento do planejamento docente?

\section{RESULTADOS E DISCUSSÕES}

Do total de vinte professores entrevistados, dezessete responderam corretamente ao questionário aplicado. Dessa forma, tentamos representar graficamente os resultados obtidos de alguns da perguntas respondidas.

A Figura 1 mostra o perfil de formação profissional dos professores entrevistados. Observa-se que $85,7 \%$ dos profissionais entrevistados possuem graduação completa, o que 
teoricamente os tornam habilitados para lecionar o ensino de Ciências de uma forma menos tradicional, pois a formação contribui para um melhor arcabouço teórico e provavelmente uma análise reflexiva de suas práticas (Alarcão, 2003).

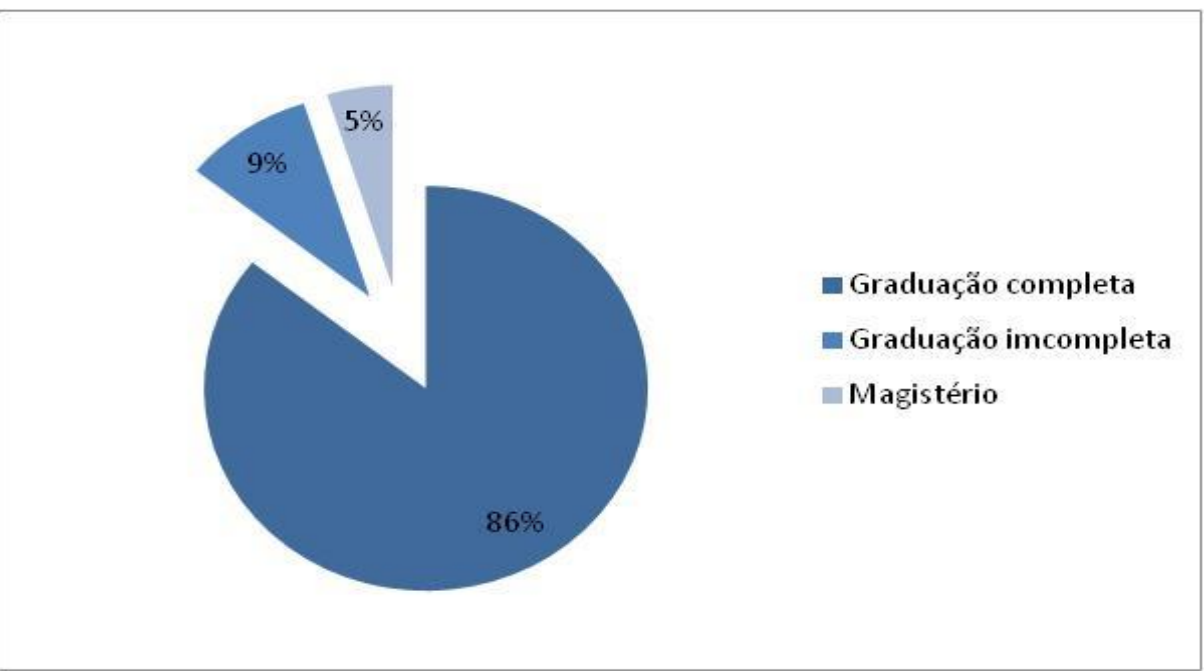

Figura 1. Formação profissional dos professores de Ciências do município de Santa Cruz/RN.

Com relação aos recursos utilizados pelos professores para o desenvolvimento das práticas de ensino (Figura 2), percebe-se a predominância do uso de metodologias de ensino tradicional pelos professores, já que todos os professores entrevistados disseram utilizar o quadro negro e livros didáticos em detrimento de aulas de campo e textos científicos. Segundo Libânio (1994), o uso de metodologias alternativas no ensino das ciências, como aulas vivenciais e textos científicos facilitam a assimilação ativa dos conhecimentos compartilhados. Em estudos anteriores, verificou-se que o uso de texto adequadamente preparado, associado à ação do professor como mediador, interferindo no processo cognitivo, e do aluno como agente ativo, possibilitou que os alunos fossem além do conhecimento de terminologias e fatos, sendo assim essencial para a aprendizagem significativa (PERTICARRARI ET AL, 2010). Ainda, alguns estudos afirmam que os textos de divulgação científica abordam temas atuais, estimulam a leitura, o questionamento e permitem a complementação do conteúdo não abordado pelo livro didático (KAWAMURA \& SALÉM, 1996; GOLDBACH ET AL, 2005. 


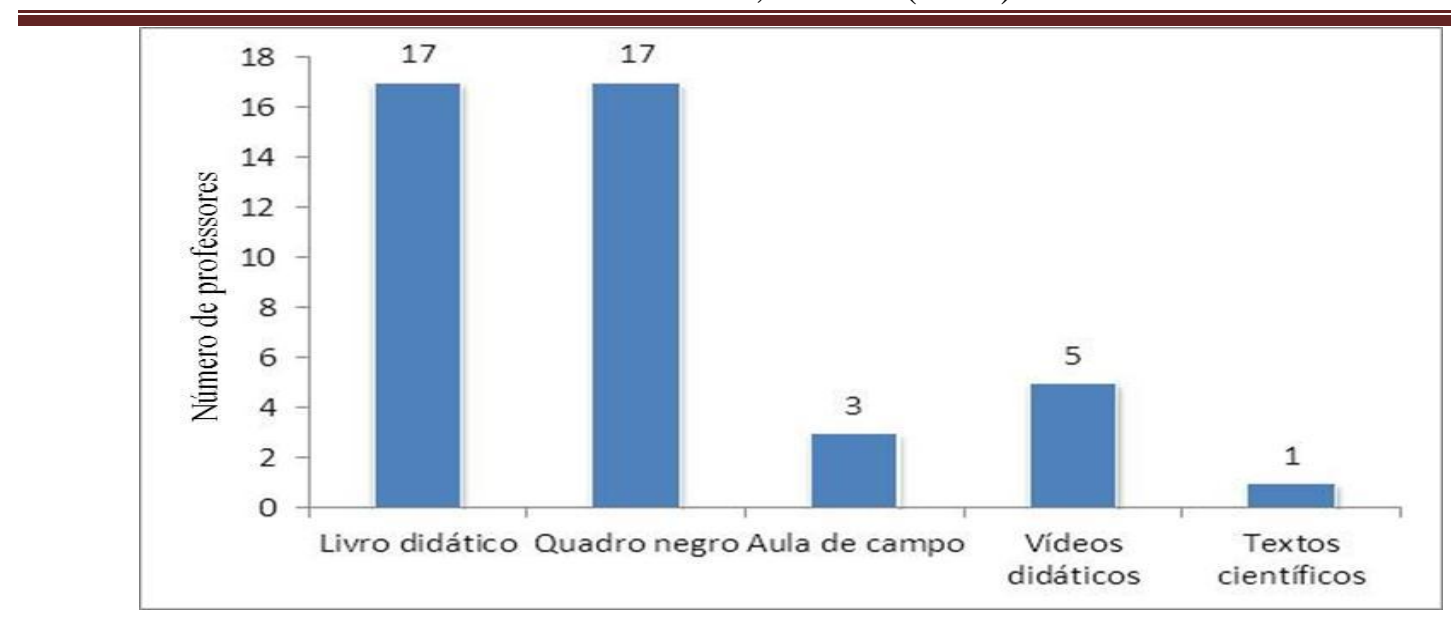

Figura 2 - Recursos utilizados pelos professores para o desenvolvimento das práticas de ensino de ciências no município de Santa Cruz/RN.

O uso do método de ensino tradicional não necessariamente é responsabilidade dos professores e de suas formações acadêmicas. Entre os fatores que contribuem para o uso do ensino tradicional está a falta de estrutura física nas escolas, como laboratórios de aulas práticas e de informática. Dentre as cinco escolas analisadas, apenas uma possui um laboratório de ciências, entretanto este se encontra inativado, já que a escola não dispõe de recursos financeiros para a sua devida manutenção.

É consenso entre os professores a importância das atividades experimentais no processo ensino-aprendizagem, já que a prática contribui com as condições de aprendizagem e desenvolvimento dos alunos (CARVALHO ET AL, 2010). Além de a estrutura física ter sido considerada um empecilho para o desenvolvimento de aulas práticas, outros fatores são levados em consideração pelos professores, como falta de tempo para o preparo e domínio do conteúdo a ser abordado (Figura 3). Fica claro nesses resultados que é extremamente viável a intervenção de outras instituições de ensino, como o IFRN (Instituto Federal de Ciência e Tecnologia do Rio Grande do Norte) nas escolas estaduais pesquisadas. Apesar dos principais fatores determinantes para o não desenvolvimento de aulas práticas serem a falta de infraestrutura escolar e tempo, é ainda significativo o número de professores que alegaram a falta de domínio acerca dos conteúdos a serem abordados. Cursos de atualização profissional voltados para a elaboração de aulas práticas podem ser ofertados em parceria com a Secretaria de Educação do município, desta forma, proporcionando aos professores a oportunidade de tornar nulas as suas necessidades em relação ao domínio dos conteúdos.

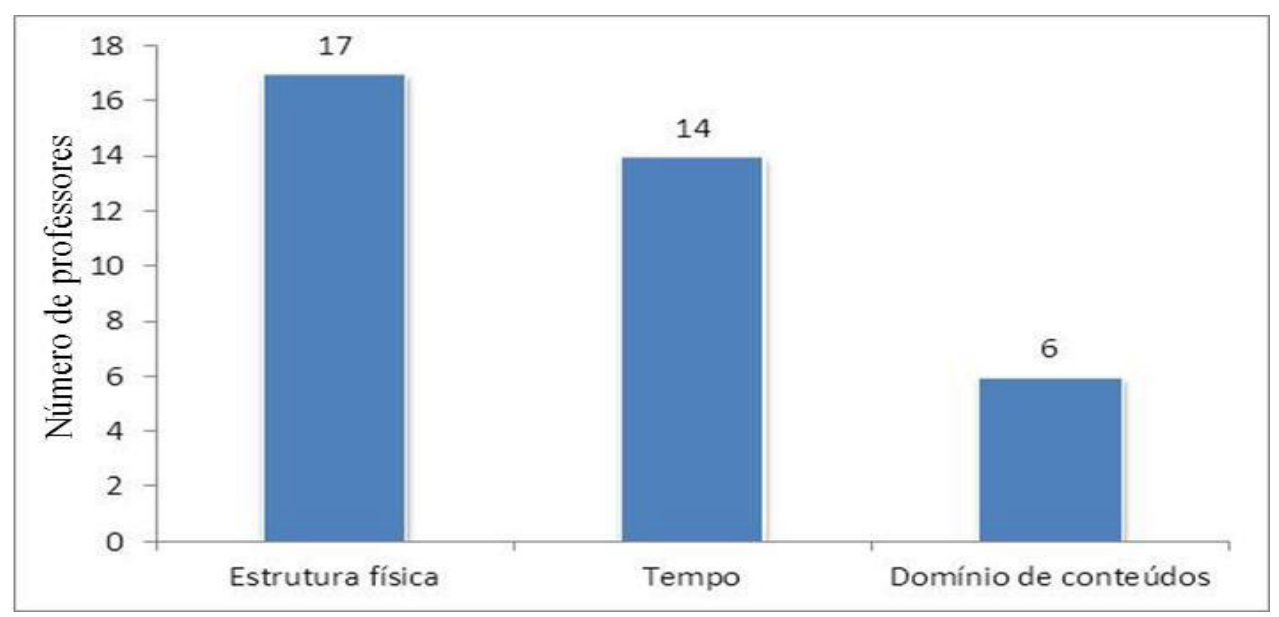

Figura 3. Principais fatores que dificultam o desenvolvimento de aulas práticas. 
Tendo em vista a grande dificuldade de professores para o desenvolvimento das aulas de ciências, nós decidimos verificar quais são as principais ferramentas utilizadas no planejamento destas (Figura 4). Pode-se concluir que todos os professores ainda utilizam como principal ferramenta os livros didáticos adotados pela escola, enquanto uma pequena parcela faz uso da internet, livros, revistas e periódicos. A utilização apenas do livro didático não parece ser suficiente para o professor trabalhar os temas mais atuais em suas aulas, já que estes se apresentam defasados por na sua maioria levarem em consideração pesquisas realizadas em torno de cinco a dez anos atrás (ROCAN \& TERZI, 1995). Ainda com relação ao uso do livro didático, PILETTI (2004) definiu:

“o professor tem pouco tempo disponível para preparar suas aulas, por isso o livro didático deve ser um recurso metodológico, fornecendo a idéia de metodologias a ser utilizadas, mas não pode "bitolar" o professor com receitas e métodos. O livro é um auxiliar mas não substitui o papel do professor."

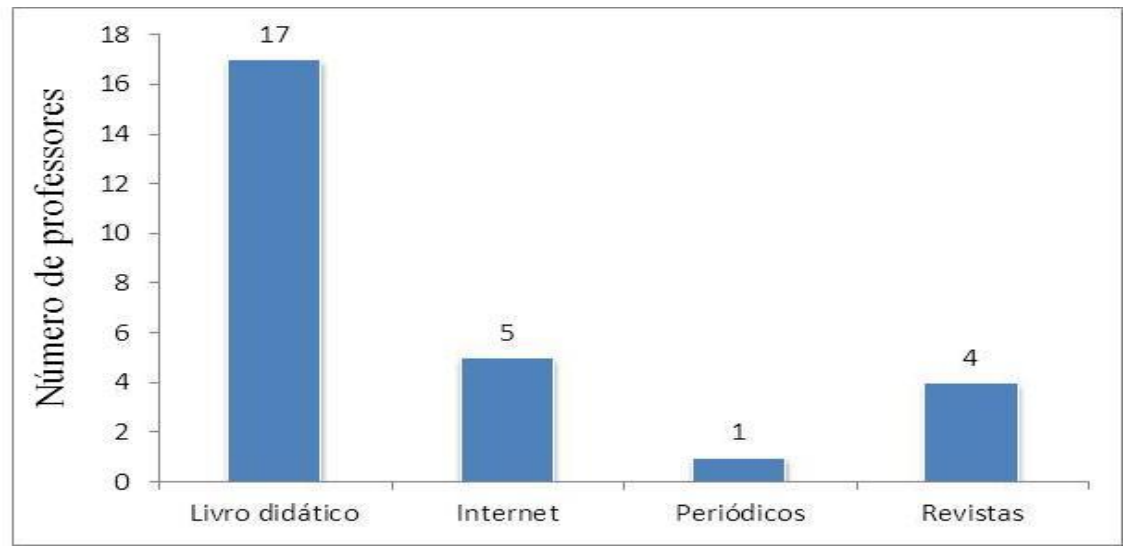

Figura 4. Ferramentas utilizadas pelos professores de Ciências de Santa Cruz/RN no planejamento didático.

Atualmente, o uso de periódicos e revistas, em conjunto com a busca de informações na internet aparece entre os principais meios de atualização docente, entretanto estas ferramentas na sua maioria são ainda inacessíveis à população (KENSKI, 2000). A introdução das novas tecnologias de informação e comunicação provocou alterações profundas na comunicação científica. A disponibilização eletrônica dos periódicos científicos teve conseqüências inevitáveis na forma de aceder ao conhecimento por parte de toda a comunidade acadêmica, bem como nos seus hábitos de pesquisa (ALMEIDA, 2006). Entretanto, nossos resultados mostraram que o uso de periódicos pelos professores é insignificante (Apenas um professor citou a utilização de periódicos no planejamento escolar - Figura 4). Portanto, fez-se necessário a indagação sobre os motivos que impedem o acesso dos professores das escolas municipais de Santa Cruz a essa ferramenta. A partir da Figura 5, podemos perceber que os principais motivos alegados pelos professores são: Falta de tempo, custo elevado e desconhecimento do uso da ferramenta. Atualmente no Brasil, o acesso e uso do Portal de Periódicos CAPES é responsável por propiciar atualização científica e tecnológica, dinamizando o processo do ensino e da pesquisa. Entretanto, o acesso ao portal ainda é restrito, inclusive na comunidade universitária, devido principalmente a fatores como: $\mathrm{O}$ excesso de informações disponíveis, as barreiras idiomáticas, a falta de instruções no manejo dos recursos, problemas em acessar fora de instituições públicas federais, a lentidão da rede e a própria falta de divulgação do Portal (MARGARIDA, 2005). Portanto, para tornar possível o acesso dos professores da rede municipal à principal ferramenta cientifica eletrônica do país, parece ser necessária a 
popularização do acesso através da disponibilidade de acesso domiciliar bem como divulgação maciça da ferramenta para este público.

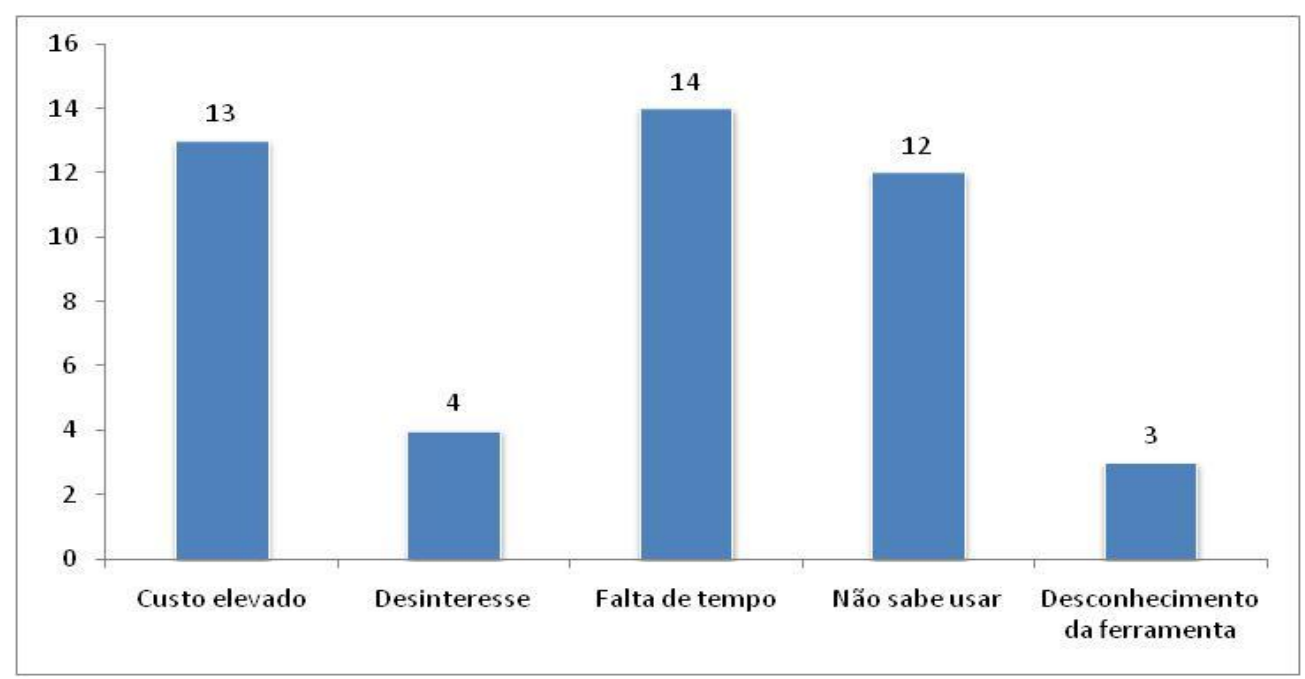

Figura 5. Principais dificuldades encontradas pelos professores para o acesso a periódicos científicos.

\section{CONCLUSÕES}

É inegável a dificuldade enfrentada pelos professores do ensino de ciências de Santa Cruz para o adequado planejamento e desenvolvimento de suas aulas. Através desta pesquisa, nós objetivamos pontuar essas dificuldades com enfoque para a prática pedagógica adotada pelos docentes. Destaca-se a falta de estrutura escolar, a falta de tempo dos professores, a dificuldade de acessos as principais tecnologias modernas (como a internet), formação profissional inespecífica para o ensino de ciências dentre outros inúmeros fatores. Os resultados encontrados neste trabalho tornam urgentes e necessários uma intervenção a fim de oferecer condições adequadas aos professores de ciências e consequentemente aos alunos, que passam a assimilar o conteúdo, tornando-se mais interessados nas questões cientificas, o que de certa forma pode contribuir no combate a um dos principais problemas da educação, a evasão escolar.

\section{AGRADECIMENTOS}

Agradecemos aos professores que se dispuseram a participar de nossa pesquisa, bem como aos diretores de escolas pelo apoio disponibilizado. O aluno Josiel Moreira da Silva agradece ao IFRN pela bolsa de pesquisa disponibilizada.

\section{REFERÊNCIAS}

1. ALARCÃO, I. Professores reflexivos em uma escola reflexiva. São Paulo: Cortez, 2003.

2. ALMEIDA, Elenara Chaves Edler - O Portal de Periódicos da Capes: estudo sobre a sua evolução e utilização. Brasília: Universidade de Brasília, Centro de Desenvolvimento Sustentável, 2006. Dissertação de Mestrado.

3. ARANHA, Maria Lúcia de Arruda. História da Educação e da Pedagogia: Geral e do Brasil. 3 ed. São Paulo: Moderna, 2006. 
4. BRASIL, Secretaria de Educação Fundamental. Parâmetros curriculares nacionais: introdução aos parâmetros curriculares nacionais. Brasília:MEC/SEF, 1997.

5. BRASIL, Secretaria de Educação Fundamental. Parâmetros curriculares nacionais: ciências naturais. Brasília: MEC/SEF, 1997.

6. CARVALHO, U. L. R.; PEREIRA, D. D.; MACEDO, E.; SILVA, K.; CIBELI, M.; FOLENA, M. A Importância Das Aulas Práticas De Biologia No Ensino Médio. $\mathrm{X}$.

7. FREIRE, Paulo. Pedagogia da autonomia. 31. ed. São Paulo: Paz e Terra, 2005.

8. GOLDBACH,T; FRIEDRICH, M.P; STELING, L.P; GANDARA, A.C.P. (2005) A utilização de artigos de divulgação científica no trabalho docente. Anais do I ENEBIO/ III EREBIO RJ/ES. Rio de Janeiro: Sociedade Brasileira de Ensino de Biologia.

9. KENSKI, Vani Moreira. Tecnologias no cotidiano: desafios para o educador. Brasília: Seed/MEC-UniRede, 2000.

10. LIBÂNIO, José Carlos. Didática. 20 ed. São Paulo: Cortez, 1994.

11. MORIN, Edgar. Os sete saberes necessários à educação do futuro. 11. ed. São Paulo: Cortez, 2005.

12. PERTICARRARI, André; TRIGO, Fernando Rossi; BARBIERI, Marisa Ramos; COVAS, Dimas Tadeu. O uso de textos de divulgação científica para o ensino de conceitos sobre ecologia a estudantes da educação básica. Ciência e Educação; v. 16, n. 2, p. 369-386, 2010.

13. PILETTI, Claudiano. Didática Especial. 15 ed. São Paulo: Ática, 2000.

14. REIS, Margarida Maria de Oliveira. Acesso e uso do Portal de Periódicos CAPES pelos professores da Universidade Federal do Acre. Universidade Federal do Acre. Florianópolis, 2005. Dissertação de mestrado.

15. RONCA, Paulo Afonso Caruso; TERZI, Cleide do Amaral. A aula operatória e a construção do conhecimento. São Paulo: Editora do Instituto Esplan, 1995.

16. SALÉM, S. \& KAWAMURA, M. (1996). O texto de divulgação e o texto didático; conhecimentos diferentes?.Anais do V Encontro de Pesquisadores em Ensino de Física. São Paulo: sociedade Brasileira de Física. 